REVIEW ARTICLE

\title{
A literature review of 2019 novel coronavirus (SARS-CoV2) infection in neonates and children
}

\author{
Matteo Di Nardo ${ }^{1}$, Grace van Leeuwen ${ }^{2}$, Alessandra Loreti ${ }^{3}$, Maria Antonietta Barbieri ${ }^{4}$, Yit Guner ${ }^{5}$, Franco Locatelli ${ }^{6}$ and \\ Vito Marco Ranieri ${ }^{7}$
}

At the time of writing, there are already millions of documented infections worldwide by the novel coronavirus 2019 (2019-nCoV or severe acute respiratory syndrome coronavirus 2 (SARS-CoV2)), with hundreds of thousands of deaths. The great majority of fatal events have been recorded in adults older than 70 years; of them, a large proportion had comorbidities. Since data regarding the epidemiologic and clinical characteristics in neonates and children developing coronavirus disease 2019 (COVID-19) are scarce and originate mainly from one country (China), we reviewed all the current literature from 1 December 2019 to 7 May 2020 to provide useful information about SARS-CoV2 viral biology, epidemiology, diagnosis, clinical features, treatment, prevention, and hospital organization for clinicians dealing with this selected population.

Pediatric Research (2021) 89:1101-1108; https://doi.org/10.1038/s41390-020-1065-5

\section{IMPACT:}

- Children usually develop a mild form of COVID-19, rarely requiring high-intensity medical treatment in pediatric intensive care unit.

- Vertical transmission is unlikely, but not completely excluded.

- Children with confirmed or suspected COVID-19 must be isolated and healthcare workers should wear appropriate protective equipment.

- Some clinical features (higher incidence of fever, vomiting and diarrhea, and a longer incubation period) are more common in children than in adults, as well as some radiologic aspects (more patchy shadow opacities on CT scan images than ground-glass opacities).

- Supportive and symptomatic treatments (oxygen therapy and antibiotics for preventing/treating bacterial coinfections) are recommended in these patients.

\section{INTRODUCTION}

Severe acute respiratory syndrome coronavirus 2 (SARS-CoV2) is the virus responsible for the coronavirus disease 2019 (COVID-19) pandemic. ${ }^{1}$ Since its first outbreak in Wuhan, in the Hubei province of China in early December 2019, ${ }^{2}$ SARS-CoV2 has spread all over the world infecting millions of people and causing hundreds of thousands o deaths [case fatality rate (CFR): $6.25 \%$, John Hopkins Coronavirus Resource Center, accessed 7 May 2020]..$^{3}$

Respiratory viral infections, in general, are more frequent and severe in children than in adults. SARS-CoV2, instead, showed a different scenario. Infection rates appear to be similar between children and adults; however, children develop a milder illness with a low CFR $(<0.1 \%) .^{3-7}$ The reasons for this milder severity in childhood are not yet understood, and the actual epidemiologic and clinical data of infected neonates and children are not sufficient to solve these gaps. Thus, due to the scarcity of data on
SARS-CoV2 in children, we aimed at evaluating the current literature available to provide useful information for clinicians dealing with this particular population.

\section{SEARCH STRATEGY}

References for this review were identified through searches on PubMED, Ovid MEDLINE, and EMBASE from 1 December 2019 to 7 May 2020, by two highly experienced librarians at Children's Hospital Bambino Gesù by using relevant terms related to 2019nCoV, COVID-19, and SARS-CoV2 in neonates and children (Supplementary Material 1). Reference lists of the articles identified by this search strategy were also searched. Earlier reports were not excluded, especially if they were highly cited articles. Only articles published in English were included in this review. Three hundred and seventy-four papers were published in PubMed, 117 in Ovid MEDLINE, and 119 in EMBASE. Among them,

\footnotetext{
${ }^{1}$ Pediatric Intensive Care Unit, Bambino Gesù Children Hospital, Rome, Italy; ${ }^{2}$ Cardiac Intensive Care Unit, Sidra Hospital, Doha, Qatar; ${ }^{3}$ Library, Bambino Gesù Children Hospital, Rome, Italy; ${ }^{4}$ Emergency Department, Bambino Gesù Children Hospital, Palidoro, Rome, Italy; ${ }^{5}$ Pediatric Surgery, Children's Hospital of Orange County, Orange, CA, USA; ${ }^{6}$ Department of Pediatric Hematology and Oncology, Bambino Gesù Children Hospital, Sapienza, University of Rome, Rome, Italy and ${ }^{7}$ Department of Medical and Surgical Science, Anesthesia and Intensive Care, Policlinico di Sant'Orsola, Alma Mater, University of Bologna, Bologna, Italy Correspondence: Matteo Di Nardo (matteo.dinardo@opbg.net)
} 


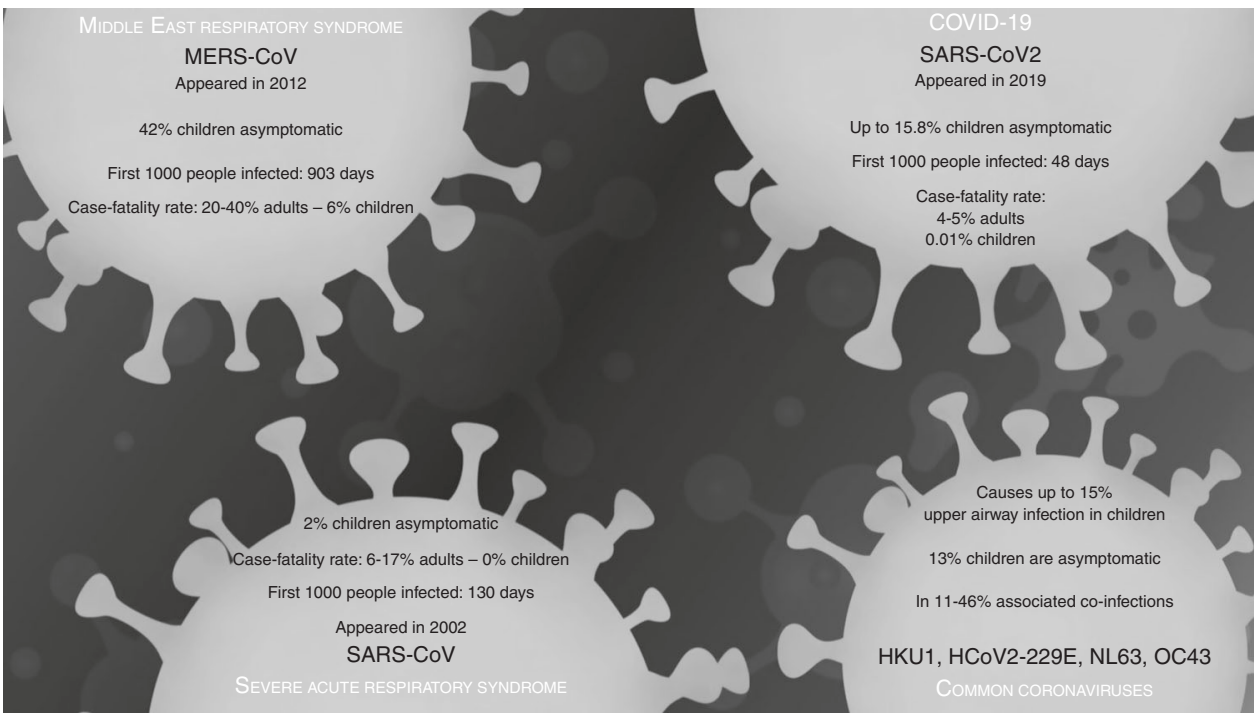

Fig. 1 Summary of coronavirus diseases (adapted from Zimmermann and Curtis ${ }^{8}$ ).

73 were deemed relevant to the purposes of this review (PRISMA flowchart Supplementary Material 2).

\section{BIOLOGICAL MECHANISMS OF VIRAL INFECTION AND LUNG INJURY}

Coronaviruses are single-strand, positive-sense RNA viruses with spike-like projections on their surface. ${ }^{8}$ These viruses can infect both animals and humans. Among human-infecting coronaviruses, four types (HKU1, NL63, 229E, and OC43) are responsible for mild forms of respiratory disease. ${ }^{9,10}$ SARS-CoV2, SARS-CoV, and the Middle East respiratory syndrome coronavirus (MERS-CoV) are zoonotic viruses and can infect humans, causing severe respiratory infections, only crossing from animals (Fig. 1).

SARS-CoV2 infects the host cells through an envelope spike (S) protein that mediates the binding and membrane fusion through the angiotensin-converting enzyme 2 (ACE-2) receptor (Fig. 2a, b). The spike protein is functionally divided into an S1 domain, responsible for receptor binding, and an S2 domain, responsible for cell membrane fusion. ${ }^{11}$ SARS-CoV2 employs the transmembrane serine protease 2 of the host cell to prime the $S$ protein and bind the ACE-2 receptor. Other transmembrane pore-forming viral proteins (viroporins) can trigger the NLRP3 (NOD-like receptor 3 inflammasome)-inducing pyroptosis in the host cell. ${ }^{12}$

ACE-2 receptors are expressed in many tissues; however, the majority are present on the alveolar epithelial type II cells. ${ }^{13}$ In addition, gene ontology enrichment analysis showed that the ACE-2-expressing epithelial cells have high levels of multiple viral process-related genes, including regulatory genes for viral processes, life cycle, assembly, and genome replication. ${ }^{13}$ All these features strongly support the hypothesis that the ACE-2 receptor mediates SARS-CoV2 replication in the lung. SARS-CoV2, through the binding to the ACE-2 receptor, downregulates the ACE-2 intracellular signaling (mitochondrial assembly receptor), causing inflammation, vasoconstriction, and fibrosis in the lung. ${ }^{13}$

\section{EPIDEMIOLOGY AND PATHOGENESIS IN NEONATES AND CHILDREN}

Published data and anecdotal reports support the notion that the number of children found to be infected by SARS-CoV2 is small and their clinical manifestations of COVID-19 are milder compared to adults. ${ }^{4-6,14-18}$
The incidence of SARS-CoV2 confirmed that pediatric cases are low and variable among countries (China: $2-12.3 \%{ }^{4,5}$ Italy: $1.2 \%{ }^{19}$ Korea: $4.8 \%,{ }^{20}$ USA: $\left.5 \%{ }^{21}\right)$. Several reasons justify this variable incidence: testing availability, testing policy ${ }^{22,23}$ (at the beginning of pandemics some countries tested only children with established contact with a person with COVID-19, then only hospitalized children with symptoms), and the fact that the infection in children is mild or without symptoms. ${ }^{24,25}$ Available data also suggest that all ages $(0-18)$ can be infected, but infants seem to be most vulnerable. ${ }^{5,26}$

Human-to-human transmission (mainly family clustered) is the major transmission mode. ${ }^{4,5,27}$ Children can be infected by inhalation of large droplets generated during coughing or sneezing or by contact with contaminated surface (fomite). ${ }^{9,10,28-30}$ As the virus can be also released in the stool, the fecal-oral transmission cannot be ruled out. ${ }^{31-34}$ Similar to SARS-CoV and MERS-CoV, nosocomial transmission of SARS-CoV2 is high, $9,10,35,36$ although no cases of nosocomial infections have been described in children during hospital recovery.

Despite the absence of clinical features of infection or positive microbiological findings in neonates born from SARS-CoV2positive mothers, ${ }^{14,18,37-42}$ vertical maternal-fetal transmission cannot be ruled out completely. ${ }^{43,44}$ Conversely, SARS-CoV2 has not been isolated from cord blood, amniotic fluid, and breast milk to date. However, it is crucial to screen pregnant women, implement strict infection control measures on those who tested positive, and monitor the neonates at risk. ${ }^{44,45}$

Since the incubation period (median 5-7 days) in children and young adolescent varies from 2 to 14 days, but is generally longer than in adults, ${ }^{10,46-48}$ dynamic observation is mandatory for suspected children. ${ }^{49,50}$ The median period from symptom onset to hospital admission for patients who were hospitalized is 2 days (1.00-3.50). Recovery generally happens in 1-2 weeks after onset. ${ }^{40,48}$ Both symptomatic patients and asymptomatic carriers can transmit SARS-CoV2. ${ }^{49,51,52}$

The basic case reproduction (R0) of SARS-CoV2 is variable (2-3.5 in the early stage of the disease); ${ }^{9}$ however, the R0 of SARS-CoV2 is higher than SARS-CoV and H1N1. ${ }^{10}$ The CFR is $\sim 6.25 \%$ (data from 7 May, John Hopkins Coronavirus Resource Center) ${ }^{3}$ and varies among countries, ${ }^{53}$ patients' age, and is influenced by testing availability. ${ }^{54}$ CFR of patients below 18 years is below $<0.1 \%$ (adapted from John Hopkins Coronavirus Resource center at 7 May 2020). ${ }^{3,7}$ 
a

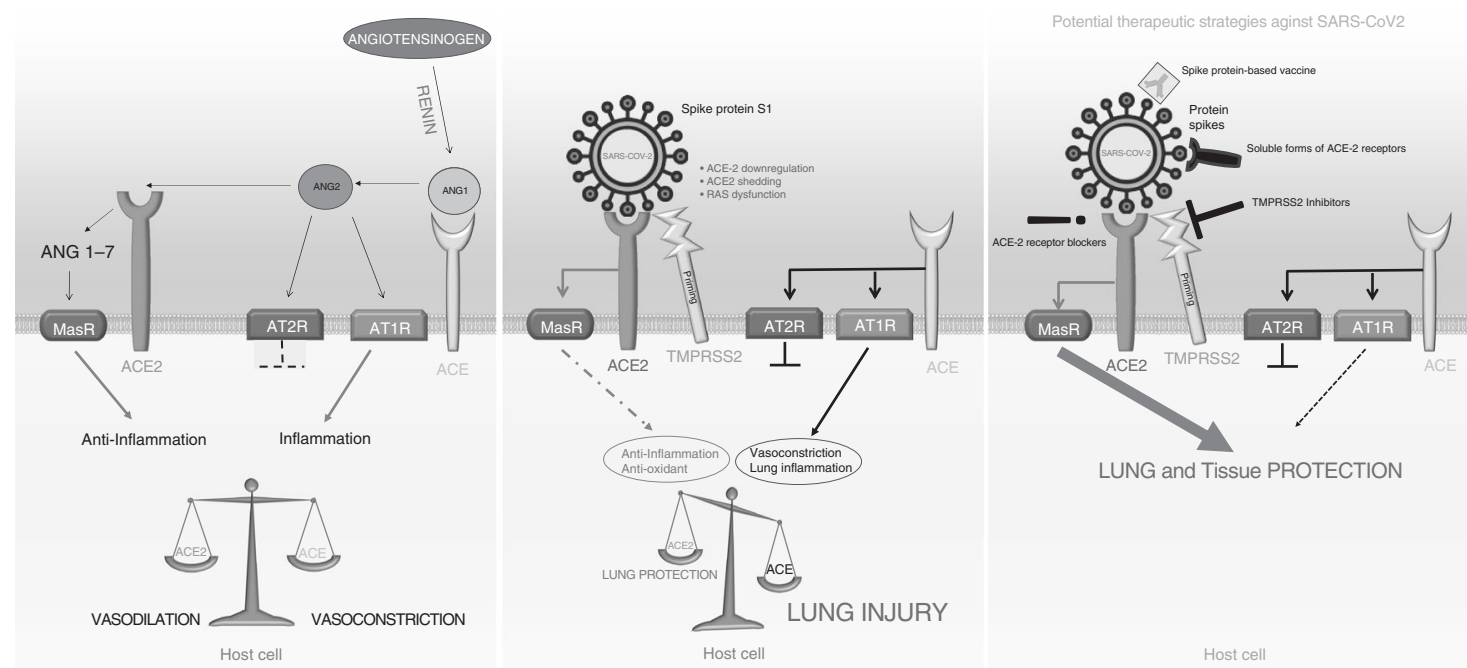

Fig. 2 Renin-Angiotensin System (RAS), Sars-CoV2 host cell entry and potential therapeutic strategies. a Renin-angiotensin system (RAS): normal physiology. Renin converts angiotensinogen in angiotensin 1 (ANG 1). Angiotensin-converting enzyme (ACE) converts ANG1 in angiotensin 2 (ANG2). Angiotensin-converting enzyme 2 (ACE-2), a homolog of ACE, is a monocarboxypeptidase that converts ANG2 into angiotensin 1-7 (ANG1-7), which, by virtue of its actions on the MasR (mitocondrial assembly receptor), opposes the molecular and cellular effects of ANG2. ANG2 promotes vasoconstriction, inflammation, and oxidative stress via the activation of AT1R (angiotensin 2 receptor 1). b SARS-CoV2 host cell entry mechanism: Spike protein (S1) binds the ACE-2 receptor once primed by the transmembrane protease serine 2 inhibitor (TMPRSS2). This binding leads to viral entry and replication and induces mechanisms of lung injury. c Potential therapeutic strategies against SARS-COV2. Spike protein-based vaccine; TMPRSS2 inhibitors to block the priming of the spike protein; surface ACE-2 receptor blocker; soluble form of ACE-2 receptor compete with the binding of SARS-CoV2 to the surface ACE-2 receptor.

This age specificity is still not completely understood. ${ }^{24,55}$ It is speculated that children, as compared with adults, may have a higher expression of ACE-2 receptors in the type II lung pneumocytes, protecting them from the severe clinical manifestation of COVID-19 (low cytokine release, low pulmonary vascular permeability, etc.). ${ }^{55}$ Other immunologic mechanisms (trained immunity, an early and high polyclonal B cell response to SARS$\mathrm{CoV} 2$ with the production of substantial numbers of plasmablasts, and an high level natural killer cells) could also contribute to explain this age-specific characteristic. ${ }^{55,56} \mathrm{~A}$ less intense mechanism of antibody-dependent enhancement, instead, could explain why COVID-19 clinical features are milder in children than in adults. $^{12}$

\section{DIAGNOSIS}

Since the World Health Organization (WHO) recently declared COVID-19 a pandemic on 11 March 2020, every patient presenting with evidence of fever, respiratory symptoms, gastrointestinal symptoms, or fatigue should be considered potentially infected (suspected case) with SARS-CoV-2.

Diagnosis of COVID-19 is made by using real-time polymerase chain reaction (RT-PCR) on samples from nasopharyngeal, oropharyngeal swabs, and lower respiratory tract samples whenever possible. ${ }^{4,5}$ Negative nasopharyngeal swab is generally re-tested after $24 \mathrm{~h}$ due to the low negative predictive value of this testing. ${ }^{57}$ SARS-CoV2 can be also detected on stools. ${ }^{33,58,59} \mathrm{~A}$ "positive" RT-PCR result reflects only the detection of viral RNA and does not necessarily indicate the presence of a viable virus. ${ }^{52}$

Confirmed cases are defined by positive molecular tests, while asymptomatic cases are defined by positive molecular tests without symptoms.

In children, more than in adults, COVID-19 poses important diagnostic challenges due to the longer incubation period that includes a prolonged interval ( $\sim-6$ days) of viral shedding prior to the onset of symptoms. ${ }^{51,60}$ Moreover, the duration of asymptomatic shedding is not only variable, but also differs according to the anatomic level (upper versus lower airways) of the infection. 49,50

At present, among adult patients in affected areas, the most common cause of viral pneumonia with unclear etiology is SARS$\mathrm{CoV} 2{ }^{2}$ conversely, in children several other pathogens (influenza, para-influenza, adenovirus, respiratory syncytial virus, metapneumovirus, or other human coronaviruses) can produce very similar clinical and radiologic findings and should be considered in the differential diagnosis. ${ }^{6,8,26,61}$ Atypical microorganisms, such as chlamydia pneumoniae and mycoplasma, must be also excluded. ${ }^{10}$

No laboratory investigations and radiological findings are diagnostic of SARS-CoV2. ${ }^{4-6,10,47,62}$

\section{CLINICAL FEATURES}

Clinical manifestations of COVID-19 in neonates and children reported are generally mild and similar among countries. ${ }^{4-6,14,16,22,23,37,38,46,63-65}$ Most commonly, at hospital admission, children presented with fever and respiratory symptoms with cough, sore throat, pharyngeal erythema, nasal congestion, tachypnea/dyspnea, and tachycardia. ${ }^{22,23,65}$ Often, gastrointestinal symptoms, including abdominal pain, nausea, vomiting, and diarrhea, were the first manifestations. ${ }^{4,5,15,46,64,66}$ Neurological manifestations such as seizures, dystonia, and altered mental status were rare. ${ }^{66}$ Neonates, instead, showed tachypnea, cough, grunting, nasal flaring, vomiting, poor feeding, diarrhea, and lethargy. ${ }^{45,61,67-69}$ Hospital admission was higher in Italy and Spain than in China and USA; ${ }^{4,21,22,65}$ however, this was mainly due to local policies (testing availability and policy, need of patient isolation) rather than clinical condition. ${ }^{22,65}$

In the largest retrospective cohort of COVID-19 pediatric patients reported so far [2134 patients including 731 (34.1\%) laboratory-confirmed and 1412 (65.9\%) suspected cases], Dong et al. ${ }^{5}$ defined the severity of COVID-19 in asymptomatic infection, mild, moderate, severe, and critical cases, based on the clinical features, laboratory testing, and X-ray imaging (Table 1). In this cohort, $4.4 \%$ of infected children were asymptomatic, while the 
Table 1. COVID-19 severity defined based on clinical features, laboratory testing, and chest X-ray imaging (adapted from Dong et al. ${ }^{5}$.

Asymptomatic

Without any clinical symptoms and signs; the chest imaging is normal, while the SARS-CoV2 nucleic acid test is positive Mild

Symptoms of acute upper respiratory tract infection, including fever, fatigue, myalgia, cough, sore throat, runny nose, and sneezing. Physical examination shows congestion of the pharynx and no auscultory abnormalities. Some cases may have no fever, or have only digestive symptoms such as nausea, vomiting, poor feeding, lethargy, abdominal pain, and diarrhea

Moderate

With pneumonia, frequent fever, and cough, mostly dry cough, followed by productive cough, some may have wheezing, but no obvious hypoxemia such as shortness of breath, and lungs can hear sputum or dry snoring and/or wet snoring. Some cases may have no clinical signs and symptoms, but chest CT shows lung lesions, which are subclinical

Severe

Early respiratory symptoms, such as fever and cough, may be accompanied by gastrointestinal symptoms such as diarrhea. The disease usually progresses $\sim 1$ week, and dyspnea occurs, with central cyanosis. Oxygen saturation is <92\%, with other hypoxia manifestations

Critical

Children can quickly progress to acute respiratory distress syndrome (ARDS) or respiratory failure, and may also have shock, encephalopathy, myocardial injury or heart failure, coagulation dysfunction, and acute kidney injury. Organ dysfunction can be life threatening

remaining children presented a mild $(50.9 \%)$ or moderate disease $(38.8 \%)$, respectively. Only $5.2 \%$ had severe disease, while $0.6 \%$ had critical disease. The proportion of severe and critical cases was $10.6 \%, 7.3 \%, 4.2 \%, 4.1 \%$, and $3.0 \%$ for the age group of $<1,1-5$, $6-10,11-15$, and $>16$ years, respectively.

Lu et al. ${ }^{4}$ showed $15.8 \%$ of COVID-19 children included in their retrospective cohort (171 SARS-CoV2 confirmed cases) were completely asymptomatic and did not show any radiological findings of pneumonia.

Respiratory coinfections were present in almost half of the cases. $^{4,5,26}$ Comorbidities, as in adult patients, ${ }^{70}$ may affect outcome $^{23}$ and the likelihood of Pediatric Intensive Care Unit (PICU) admission. ${ }^{4,23}$

In adults, the incidence of ICU admission was high and variable among countries (5\% in China and $9 \%$ in Italy) ${ }^{70,71}$ in children, the incidence was lower (0.21-5.2\% among Chinese PICUs, ${ }^{4,5,15} 0.04 \%$ in $U_{S A}{ }^{23}$ ). Of note, several biases (retrospective nature of these studies, 5,61 the proportion of the detected cases, the use of different PICU admission criteria among centers, ${ }^{5}$ the use of the same data source with overlapping data-Chinese Centers for Disease Control and Prevention database-and the high number of suspected cases ${ }^{47}$ ) could have affected the interpretation of these results.

Most of the laboratory abnormalities in children with COVID-19 are nonspecific. Henry et al. ${ }^{62}$ reviewed the data of 66 children from 12 different studies and found that $69.2 \%$ of children had normal leukocyte counts and that neutrophilia or neutropenia were rare $(<5 \%)$. Platelet count was variable among studies (generally higher than the normal range), while C-reactive protein and procalcitonin were increased in $13.6 \%$ and $10.6 \%$ of the cases, respectively. ${ }^{62}$

Children admitted to the $\mathrm{PICU}^{15}$ showed normal or increased whole blood counts (7/8) and increased C-reactive protein, procalcitonin, and lactate dehydrogenase (6/8). High levels of pro-inflammatory and anti-inflammatory cytokines were also present similarly to the adult patients. ${ }^{72,73}$

Although lymphocytopenia is very common in adults with severe COVID-19 and associated with worse outcomes, ${ }^{47}$ it is less common in children (2-3.5\%), likely due to the constitutional high percentage of lymphocytes typical of this age. ${ }^{62,74}$ In adult patients, high ferritin, high D-dimers, and coagulopathy were associated with poor prognosis, ${ }^{70}$ but these laboratory findings were rare in children; high D-dimers levels were found in one of the two patients who died from COVID-19., ${ }^{45}$ However, during April 2020, a surge of anecdotal cases showing a hyperinflammatory state (pediatric multisystem inflammatory syndrome temporally associated with COVID-19) and features similar to atypical Kawasaki disease or Kawasaki disease shock syndrome were reported in Europe (United Kingdom, Spain, Italy). ${ }^{75,76}$ Many of these patients had positive SARS-CoV2 antibodies and presented an inflammatory state (elevated concentration of Creactive protein, procalcitonin, ferritin triglycerides, and $D$-dimers) with cutaneous rash, peripheral edema, conjunctivitis, myocardial dysfunction (elevated cardiac enzymes), and coronary vessels inflammation.

Radiologic findings of SARS-CoV2 viral pneumonia were also variable among children (Fig. 3). ${ }^{4}$ At hospital admission, many children presented a chest X-ray showing an interstitial pneumo$\mathrm{nia},{ }^{26}$ while chest computed tomography (CT) scan showed patchy shadows (unilateral and bilateral) with opacities of high density. The typical adult feature of ground-glass opacity was less frequent at hospital admission $(32.7 \%){ }_{i}^{4}$ instead, it was more common in patients admitted to the PICU for respiratory failure. ${ }^{4-6,26,77-79}$ Bedside lung ultrasonography was also used as a diagnostic tool in the emergency departments in a minority of patients; ${ }^{80} 90 \%$ of these received a diagnosis of interstitial lung syndrome without further radiographic imaging. ${ }^{65}$

\section{TREATMENT}

Treatment of COVID-19 in neonates and children mainly relies on supportive care. ${ }^{4,10}$

Home isolation is the first step to manage children with mild symptoms and no underlying chronic conditions. Hospitalization may be considered if rapid deterioration is anticipated or if the patient is not able to urgently return to hospital when signs and symptoms of complicated disease arise. Moderate cases should be managed in hospital, monitoring vital signs and oxygen saturation. Supportive care for these children includes temperature control with antipyretics, bed rest, hydration, and good nutrition. Routine antibiotics and antifungal drugs must be avoided and used only when coinfections are proven or strongly suspected. ${ }^{10,15}$

In hypoxic patients, oxygen therapy should be immediately initiated. ${ }^{81}$ Several devices [low flow nasal cannula, high-flow nasal cannula (HFNC), and noninvasive ventilation (NIV)] can be used according to the centers' experience. Caution must be taken, since all noninvasive techniques bear the risk of aerosol contamination; strict personal protection equipment (PPE) must be used when caring for these patients.

Invasive mechanical ventilation is indicated if: $\mathrm{SpO}_{2} / \mathrm{FiO}_{2}<221$ or if there is no improvement in oxygenation (target $\mathrm{SpO}_{2} 92-97 \%$ with $\mathrm{FiO}_{2}<0.4$ ) within $30-60 \mathrm{~min}$ of $\mathrm{HFNC}$ or if there is no 

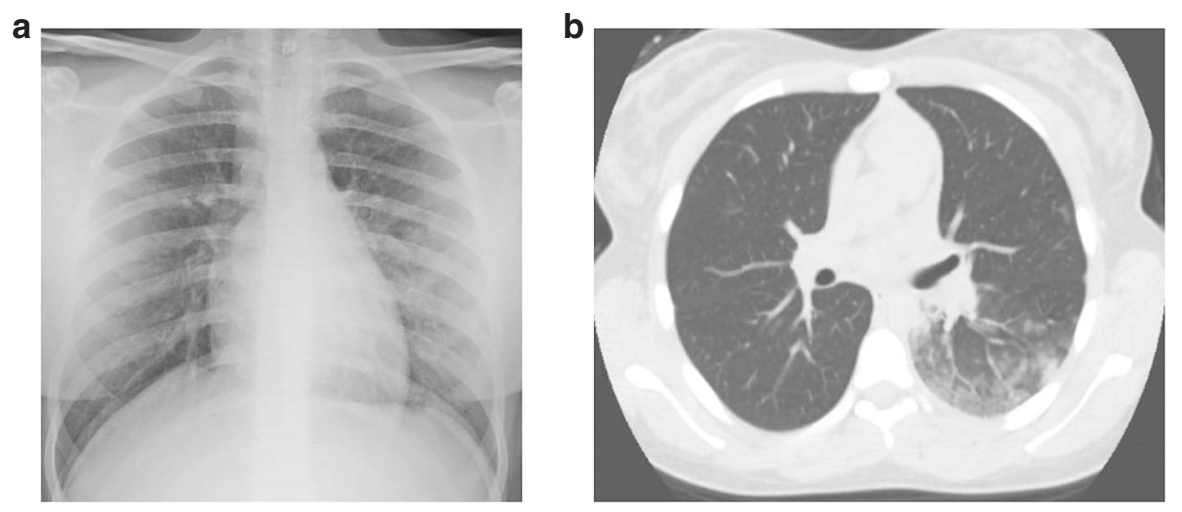

Fig. 3 Chest X-ray (interstitial pneumonia) and chest computed tomography (left patchy shadow opacities of high density) of a 16-yearold patient presenting persisting fever $\left(38.8^{\circ} \mathrm{C}\right)$, cough, and myalgia (hospital admission). a Chest $\mathrm{X}$ ray and $\mathbf{b}$ chest computed tomography. Vital signs: respiratory rate 22 breaths $/ \mathrm{min}, \mathrm{SpO}_{2}: 97 \%$ in room air. The patient was supported with high-flow nasal cannula $25 \mathrm{~L} /$ $\min , \mathrm{FiO}_{2}: 30 \%$ in the pediatric ward.

improvement in oxygenation (target $\mathrm{SpO}_{2} 92-97 \%$ and $\mathrm{FiO}_{2}<0.6$ ) within $60-90$ min of CPAP/NIV. ${ }^{81}$ Escalating therapies are recommended in case of refractory hypoxia (surfactant therapy in neonates, inhaled nitric oxide, high frequency oscillatory ventilation, and extracorporeal membrane oxygenation). ${ }^{81-83}$

A small portion of children with COVID-19 developed septic shock; ${ }^{5,15,84}$ thus, this condition must be always suspected and managed according to the current pediatric guidelines since specific issues for COVID-19 have not been reported so far. ${ }^{85}$ Corticosteroids should not be used in pediatric patients, ${ }^{86}$ except when required for other indications, such as asthma exacerbations, refractory shock, or evidence of cytokine storm. ${ }^{16}$

Several treatment options (intravenous immunoglobulin, interleukin-1 (IL-1) blockade, IL-6 receptor blockade, azythromycin-chloroquine, plasma exchange, infusion of plasma from convalescent subjects, cytokine adsorption filters) have been used in critically ill adult patients; however, data on their efficacy and safety have not been reported yet, thus caution should be used also in children. ${ }^{87}$

Antiviral drugs should be used with caution after weighing advantages and disadvantages. For those with mild symptoms, low dosage of interferon-a nebulization has been used $^{16}$ in combination with oral ribavirin. Lopinavir/litonavir ${ }^{15}$ and remdesivir $^{88,89}$ have been used in more severe cases; however, their efficacy and safety in children remain to be determined. ${ }^{90}$ Remdesivir should be preferred in children because of its positive effects in a recent adult trial; ${ }^{88,89}$ however, when not available, or when patients are not good candidate to remdesivir, hydroxychloroquine could be considered. ${ }^{88}$ The combination of three or more antiviral drugs is generally not recommended. ${ }^{90}$

Potential therapeutic strategies for SARS-COV2 are the spike protein-based vaccine, the inhibitors of transmembrane protease serine 2 activity, and the delivery of excessive soluble form of ACE2 or antibody against the surface of ACE-2 receptors (Fig. 2c). ${ }^{13}$

\section{PREVENTION AND HEALTHCARE ORGANIZATION}

COVID-19 has no approved treatment in neonates and children and a large-scale vaccine is still under development; thus, prevention is crucial. ${ }^{10,91}$

SARS-CoV-2 has unique characteristics that makes its prevention complex. SARS-CoV-2 can cause an asymptomatic infection, can be transmitted during the incubation period and after clinical recovery, ${ }^{13}$ has a very high affinity to ACE-2 receptors, which are expressed on many mucosal surfaces, resulting in high transmissibility, and can be spread also by fomite. ${ }^{10}$

The high transmissibility and low CFR, combined with the discouraging projections of the spread of the virus among adults, ${ }^{70}$ fostered many governments, at the beginning of March 2020, to adopt stringent containment and self-isolation measures to reduce the spread of the virus. An intense public health response was started by many countries after the pandemic declaration and involved many strategies: lockdown of the cities and mass quarantine, social distancing mandates, schools closure, cancellation of public gatherings, reduction of domestic and international flights, development of environmental measures and personal protection procedures, and strict contacts tracings by the medical and public health professionals. These measures aim to delay major surges of patients and to lower the demand for hospital extra beds, while protecting the most vulnerable subjects from infection, especially the elderly and those with comorbidities. ${ }^{92}$

Data showed that pediatric cases requiring high-intensity medical assistance are uncommon; ${ }^{5,15}$ however, isolation of all suspected and confirmed patients remains mandatory to avoid the spread of SARS-CoV2 among caregivers and healthcare workers. Therefore, many pediatric hospitals have developed local guidelines and logistic plans (simulations and training courses, reduction of elective surgeries and visits to outpatient clinics, etc.) to identify in advance potential surge capacity in the form of dedicated environment with extra beds for isolation, quarantine, and dedicated staff. As stocks of PPE might run low during a period of pandemic, strict hospital policies should also be adopted according to the WHO guidelines. ${ }^{93}$ Furthermore, considering the high number of adult ICU admissions and the difficulties associated to create extra beds in a short period of time, ${ }^{70}$ pediatric intensivists and nurses should be ready and prepared to offer help by managing adult patients in $\mathrm{PICU}^{94}$ or to help in adult ICUs.

Differently from adults, home isolation is not easily performed in children, because they often require the presence of the parents, limiting the use of protective distances $(>1.5 \mathrm{~m})$. In those cases, all people sharing a common environment with a SARSCoV2-positive child should consider the use of gloves and face masks, if available. Hand hygiene practices are extremely important to prevent the spread of the COVID-19 virus at home and in public environments. The WHO recommends washing hands, especially after coughing or sneezing (including sneeze/ cough into elbow or tissue), before eating and after using the toilet or sharing common spaces. ${ }^{95}$ Hand washing also interrupts transmission of other viruses and bacteria causing common colds, flu, and pneumonia, thus reducing the general burden of disease. Relatives at risk (e.g., people over the age of 65 years, pregnant women, people who are immunocompromised or who have chronic heart, lung, or kidney conditions) ${ }^{96}$ should be isolated in protected environment, avoiding exposures to infected children. Because infants cannot wear masks, parents must wear masks, 

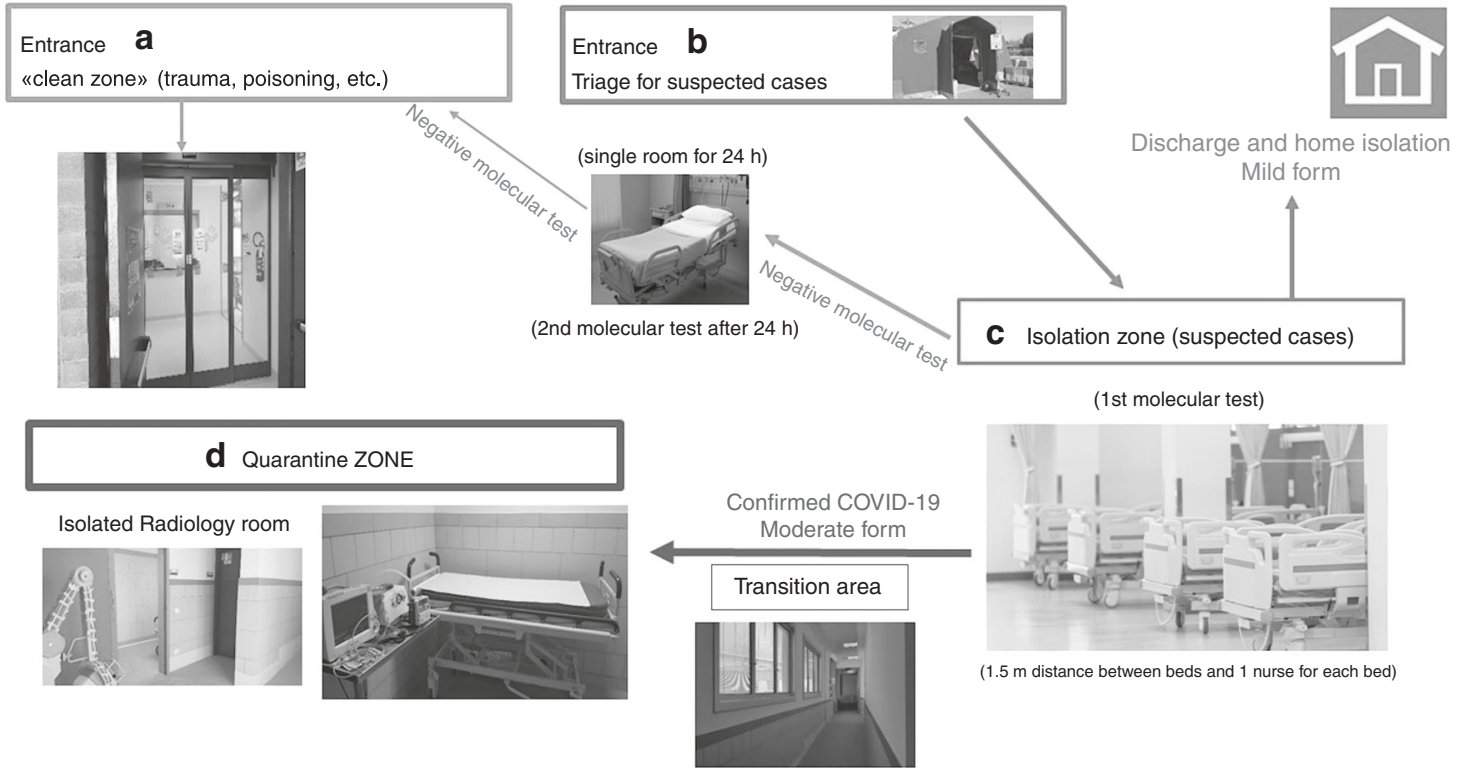

Fig. 4 Enhanced traffic control system during COVID-19 pandemic. Enhanced traffic control system used in Children's Hospital Bambino Gesù, Rome, Italy.

wash hands before close contacts, and sterilize the toys and tablet regularly. ${ }^{97}$

All suspected children requiring hospital assistance must be isolated in single rooms (whenever possible, or in dedicated environments, maintaining adequate distances between beds) until the results of the test are available; confirmed patients must be placed in dedicated area for quarantine. A dedicated algorithm must be adopted for the use of the operating theaters in suspected or confirmed COVID-19 cases, according to the urgency of the operation, anticipated viral burden at the surgical site, and the risk that a procedure could spread the virus by aereosol. ${ }^{98,99}$ Negative pressure rooms are of help, but not mandatory to manage these patients. ${ }^{10}$ All rooms and transition environments must be decontaminated after the patient discharge (Fig. 3).

Since a high number of health care workers has been infected by SARS-CoV2, all suspected patients, until proven negative, must be assisted by health care providers using $\mathrm{PPE}^{93}$ and all aerosol generating procedures (intubation, bronchoscopy, tube/tracheostomy suctioning, etc.) must be also performed using airborne transmission precautions. ${ }^{93}$

Enhanced traffic control bundling strategies must be adopted by all emergency departments, ${ }^{100}$ including a triage zone, transition zones conduction to a quarantine ward or to an isolation ward (Fig. 4). A dedicated pathway for children nonSARS-CoV2 suspected (e.g., trauma, poisoning, etc.) must also be created in parallel to avoid contact. Telemedicine should be implemented to help reduce hospital and clinic visits, ${ }^{101,102}$ by triaging low-acuity patients while delivering high-quality care. ${ }^{103}$

The scarcity of pediatric cases and the current literature on the topic, as well as the absence of high-quality evidence-based guidelines, has led pediatricians to share experiences and personal communication via online meetings and open access medical education channels. The use of webinars and communication about newly released papers on social media channels such as Twitter, Telegram and WhatsApp, greatly improved the dissemination of knowledge among health care providers.

\section{CONCLUSION}

At the time of this review (7 May 2020), SARS-CoV2 has infected millions of people in the world and caused hundreds of thousands confirmed deaths, but data regarding the epidemiologic and clinical characteristics in neonates and children are still scarce. The purpose of this review was to evaluate the current literature that includes neonates and children to date, providing useful information for clinicians dealing with this selected population. The earliest epidemiologic data show that SARS-CoV2 has a dominant family-cluster transmission and that children present a mild form of COVID-19 (CFR: $<0.1 \%$ ), rarely requiring high-intensity medical treatment in PICU. Vertical transmission is unlikely, but not completely excluded. Diagnosis is performed primarily via molecular nucleic acid amplification testing. Patients with confirmed or suspected COVID-19 should be isolated and healthcare workers should wear appropriate protective equipment. Some clinical features (higher incidence of fever, vomiting and diarrhea, and a longer incubation period) are more common in children than in adults, as well as some radiologic aspects, including the presence of patchy shadow opacities on CT scan images. Treatment options are extrapolated from adult data. Thus, supportive and symptomatic treatments (oxygen therapy and antibiotics for bacterial coinfections) are recommended in these patients. More studies on neonates and children are needed to address these gaps and to provide more robust recommendations to manage COVID-19.

\section{AUTHOR CONTRIBUTIONS}

Each author made a substantial contribution to this review and met the Pediatric Research authorship requirements. M.D.N., G.V.L., and A.L. contributed to the review design, data acquisition, and screening. M.D.N., M.A.B., and Y.G. contributed to the interpretation of the data and article drafting. M.D.N., F.L., and V.M.R. contributed to the article drafting and revisions. All authors have approved the final manuscript.

\section{ADDITIONAL INFORMATION}

The online version of this article (https://doi.org/10.1038/s41390-020-1065-5) contains supplementary material, which is available to authorized users.

Competing interests: The authors declare no competing interests.

Publisher's note Springer Nature remains neutral with regard to jurisdictional claims in published maps and institutional affiliations. 


\section{REFERENCES}

1. Lai, C. C., Shih, T. P., Ko, W. C., Tang, H. J. \& Hsueh, P. R. Severe acute respiratory syndrome coronavirus 2 (SARS-CoV-2) and coronavirus disease-2019 (COVID-19): the epidemic and the challenges. Int. J. Antimicrob. Agents 55, 105924 (2020).

2. Zhu, N. et al. A novel coronavirus from patients with pneumonia in China, 2019. N. Engl. J. Med. 382, 727-733 (2020).

3. Center for Systems Science and Engineering (CSSE) at JHU. COVID-19 Dashboard. https://coronavirus.jhu.edu/map.

4. Lu, X. et al. SARS-CoV-2 infection in children. N. Engl. J. Med. 382, 1663-1665 (2020).

5. Dong, Y. et al. Epidemiology of COVID-19 among children in China. Pediatrics 145, e20200702 (2020).

6. Liu, W. et al. Detection of Covid-19 in children in early January 2020 in Wuhan. China. N. Engl. J. Med. 382, 1370-1371 (2020).

7. Jeng, M. J. COVID-19 in children: current status. J. Chin. Med. Assoc. 83, 527-533 (2020).

8. Zimmerman, P. \& Curtis, N. Coronavirus infections in children including COVID19: an overview of the epidemiology, clinical features, diagnosis, treatment and prevention options in children. Pediatr. Infect. Dis. J. 39, 355-368 (2020).

9. Wang, Y., Wang, Y., Chen, Y. \& Qin, Q. Unique epidemiological and clinical features of the emerging 2019 novel coronavirus pneumonia (COVID-19) implicate special control measures. J. Med. Virol. 92, 568-576 (2020).

10. Singhal, T. A Review of Coronavirus Disease-2019 (COVID-19). Indian J Pediatr 87 281-286 (2020).

11. Lu, R. et al. Genomic characterisation and epidemiology of 2019 novel coronavirus: implications for virus origins and receptor binding. Lancet 395, 565574 (2020).

12. Fu, Y., Cheng, Y. \& Wu, Y. Understanding SARS-CoV-2-mediated inflammatory responses: from mechanisms to potential therapeutic tools. Virol. Si. 35, 266-271 (2020).

13. Zhang, H., Penninger, J. M., Li, Y., Zhong, N. \& Slutsky, A. S. Angiotensin-converting enzyme 2 (ACE2) as a SARS-CoV-2 receptor: molecular mechanisms and potential therapeutic target. Intensive Care Med. 46, 586-590 (2020).

14. Cai, J. et al. A case series of children with 2019 novel coronavirus infection: clinical and epidemiological features. Clin. Infect. Dis. Feb;ciaa198 (2020).

15. Sun, D. et al. Clinical features of severe pediatric patients with coronavirus disease 2019 in Wuhan: a single center's observational study. World J. Pediatr. 16, 251-259 (2020).

16. Shen, K. et al. Diagnosis, treatment, and prevention of 2019 novel coronavirus infection in children: experts' consensus statement. World J. Pediatr. 16, 223-231 (2020).

17. Stower, $\mathrm{H}$. Clinical and epidemiological characteristics of children with COVID19. Nat. Med. 26, 465 (2020)

18. Yang, P. et al. Clinical characteristics and risk assessment of newborns born to mothers with COVID-19. J. Clin. Virol. 127, 104356 (2020).

19. Livingston E., Bucher K. Coronavirus Disease 2019 (COVID-19) in Italy. Jama (2020).

20. Korean Society of Infectious Diseases. Report on the Epidemiological Features of Coronavirus Disease 2019 (COVID-19) Outbreak in the Republic of Korea from January 19 to March 2, 2020. J. Korean Med. Sci. 35, e112 (2020).

21. CDC COVID-19 Response Team. Coronavirus Disease 2019 in Children - United States, February 12-April 2, 2020. MMWR Morb Mortal Wkly Rep 2020;69:422426. https://doi.org/10.15585/mmwr.mm6914e44e4.

22. Tagarro, A. et al. Screening and severity of coronavirus disease 2019 (COVID-19) in children in Madrid, Spain. JAMA Pediatr. 8, e201346 (2020).

23. Pathak, E. B., Salemi, J. L., Sobers, N., Menard, J. \& Hambleton, I. R. COVID-19 in Children in the United States: intensive care admissions, estimated total infected, and projected numbers of severe pediatric cases in 2020. J. Public Health Manag. Pract. 26, 325-333 (2020).

24. Brodin, P. Why is COVID-19 so mild in children? Acta Paediatr. 109, 1082-1083 (2020).

25. Lee, P. I., Hu, Y. L., Chen, P. Y., Huang, Y. C. \& Hsueh, P. R. Are children less susceptible to COVID-19? J. Microbiol. Immunol. Infect. 53, 371-372 (2020).

26. Xia, W. et al. Clinical and CT features in pediatric patients with COVID-19 infection: different points from adults. Pediatr. Pulmonol. 55, 1169-1174 (2020).

27. Su, L. et al. The different clinical characteristics of corona virus disease cases between children and their families in China - the character of children with COVID-19. Emerg Microbes Infect 9, 707-713 (2020).

28. Cheng, Z. J. \& Shan, J. 2019 Novel coronavirus: where we are and what we know. Infection 48, 155-163 (2020).

29. van Doremalen, N. et al. Aerosol and surface stability of SARS-CoV-2 as compared with SARS-CoV-1. N. Engl. J. Med. 382, 1564-1567 (2020).

30. Yung, C. F. et al. Environment and personal protective equipment tests for SARSCoV-2 in the isolation room of an infant with infection. Ann. Intern. Med. M20$0942(2020)$
31. $\mathrm{Ma}, \mathrm{X}$. et al. Do children need a longer time to shed SARS-CoV-2 in stool than adults? J. Microbiol. Immunol. Infect. 53, 373-376 (2020).

32. Yeo, C., Kaushal, S. \& Yeo, D. Enteric involvement of coronaviruses: is faecal-oral transmission of SARS-CoV-2 possible? Lancet Gastroenterol Hepatol 5, 335-337 (2020).

33. Tian, Y., Rong, L., Nian, W. \& He, Y. Review article: gastrointestinal features in COVID-19 and the possibility of faecal transmission. Aliment. Pharmacol. Ther. 51, 843-851 (2020).

34. Dona, D., Minotti, C., Costenaro, P., Da Dalt, L. \& Giaquinto, C. Fecal-oral transmission of Sars-Cov-2 in children: is it time to change our approach? Pediatr. Infect. Dis. J. 39, e133-e134 (2020).

35. Bartoszko, J. J., Farooqi, M. A. M., Alhazzani, W. \& Loeb, M. Medical masks vs N95 respirators for preventing COVID-19 in health care workers. A systematic review and meta-analysis of randomized trials. Influenza Other Respir. Viruses 14, 365-373 (2020).

36. Ferioli, M. et al. Protecting healthcare workers from SARS-CoV-2 infection: practical indications. Eur. Respir. Rev. 29, 200068 (2020).

37. Zhu, H. et al. Clinical analysis of 10 neonates born to mothers with 2019-nCoV pneumonia. Transl Pediatr. 9, 51-60 (2020).

38. Chen, $\mathrm{H}$. et al. Clinical characteristics and intrauterine vertical transmission potential of COVID-19 infection in nine pregnant women: a retrospective review of medical records. Lancet 395, 809-815 (2020).

39. Schwartz, D. A. An analysis of 38 pregnant women with COVID-19, their newborn infants, and maternal-fetal transmission of SARS-CoV-2: maternal coronavirus infections and pregnancy outcomes. Arch. Pathol. Lab. Med. https://doi. org/10.5858/arpa.2020-0901-SA (2020).

40. Hong, H., Wang, Y., Chung, H. T. \& Chen, C. J. Clinical characteristics of novel coronavirus disease 2019 (COVID-19) in newborns, infants and children. Pediatr. Neonatol. 61, 131-132 (2020).

41. Li, Y. et al. Lack of vertical transmission of severe acute respiratory syndrome coronavirus 2. China. Emerg. Infect. Dis. 26, 1335-1336 (2020).

42. Zeng, $\mathrm{H}$. et al. Antibodies in infants born to mothers with COVID-19 pneumonia. JAMA 16, 223-231 (2020).

43. Zeng, L. et al. Neonatal early-onset infection with SARS-CoV-2 in 33 neonates born to mothers with COVID-19 in Wuhan, China. JAMA Pediatr. 174, 722-725 (2020).

44. Buonsenso, D. et al. Neonatal late onset infection with severe acute respiratory syndrome coronavirus 2. Am. J. Perinatol. 37, 869-872 (2020).

45. Wang, L. et al. Chinese expert consensus on the perinatal and neonatal management for the prevention and control of the 2019 novel coronavirus infection (First edition. Ann. Transl. Med. 8, 47 (2020).

46. Wang, X. F. et al. [Retracted: Clinical and epidemiological characteristics of 34 children with 2019 novel coronavirus infection in Shenzhen]. Zhonghua Er Ke Za Zhi 58, E008 (2020).

47. Ludvigsson, J. F. Systematic review of COVID-19 in children shows milder cases and a better prognosis than adults. Acta Paediatr. 109, 1088-1095 (2020).

48. Cao, Q., Chen, Y. C., Chen, C. L. \& Chiu, C. H. SARS-CoV-2 infection in children Transmission dynamics and clinical characteristics. J. Formos. Med. Assoc. 119, 670-673 (2020).

49. Kam, K. Q. et al. A well infant with coronavirus disease 2019 (COVID-19) with high viral load. Clin. Infect. Dis. ciaa201 (2020).

50. Lai, C. C. et al. Asymptomatic carrier state, acute respiratory disease, and pneumonia due to severe acute respiratory syndrome coronavirus 2 (SARS-CoV2): facts and myths. J. Microbiol. Immunol. Infect. 53, 404-412 (2020).

51. He, X. et al. Temporal dynamics in viral shedding and transmissibility of COVID19. Nat. Med. 26, 672-675 (2020).

52. Wolfel, R. et al. Virological assessment of hospitalized patients with COVID-2019. Nature 581, 465-469 (2020).

53. Streeck, H., Hartmann, G., Exner, M., \& Schmid, M. Vorläufiges Ergebnis und Schlussfolgerungen der COVID-19 Case-Cluster-Study (Gemeinde Gangelt). https://www.land.nrw/sites/default/files/asset/document/zwischenergebnis_ covid19_case_study_gangelt_0.pdf.

54. Bendavid, E. et al. COVID-19 Antibody Seroprevalence in Santa Clara County, California. medRxiv 2020.04.14.20062463. https://doi.org/10.1101/ 2020.04.14.20062463.

55. Cristiani, L. et al. Will children reveal their secret? The coronavirus dilemma. Eur Respir. J. 55, 2001617 (2020).

56. Carsetti, R. Q. C. et al. The immune system of children: the key to understanding SARS-CoV-2 susceptibility? Lancet Child Adolesc. Health 4, 414-416 (2020).

57. Chu, D. K. W. et al. Molecular Diagnosis of a Novel Coronavirus (2019-nCoV) Causing an Outbreak of Pneumonia. Clin. Chem. 66, 549-555 (2020).

58. Zhang, T. et al. Detectable SARS-CoV-2 viral RNA in feces of three children during recovery period of COVID-19 pneumonia. J. Med. Virol. 92, 909-914 (2020).

59. Xing, Y. H. et al. Prolonged viral shedding in feces of pediatric patients with coronavirus disease 2019. J. Microbiol. Immunol. Infect. 53, 473-480 (2020). 
60. Lu, Y. et al. Symptomatic infection is associated with prolonged duration of viral shedding in mild coronavirus disease 2019: a retrospective study of 110 children in Wuhan. Pediatr. Infect. Dis. J. 39, e95-e99 (2020).

61. Lu, Q. \& Shi, Y. Coronavirus disease (COVID-19) and neonate: What neonatologist need to know. J. Med. Virol. 92, 564-567 (2020).

62. Henry, B. M., Lippi, G. \& Plebani, M. Laboratory abnormalities in children with novel coronavirus disease 2019. Clin. Chem. Lab. Med. 58, 1135-1138 (2020).

63. Yang, P., Liu, P., Li, D. \& Zhao, D. Corona virus disease 2019, a growing threat to children? J. Infect. 80, 671-693 (2020).

64. Zheng, F. et al. Clinical characteristics of children with coronavirus disease 2019 in Hubei. China. Curr. Med. Sci. 40(Apr), 275-280 (2020).

65. Parri, N., Lenge, M. \& Buonsenso, D. Children with Covid-19 in pediatric emergency departments in Italy. N. Engl. J. Med. 383, 187-190 (2020).

66. Dugue, R. et al. Neurologic manifestations in an infant with COVID-19. Neurology 94, 1100-1102 (2020)

67. De Luca, D. Managing neonates with respiratory failure due to SARS-CoV-2. Lancet Child Adolesc. Health 4, e8 (2020).

68. Li, F., Feng, Z. C. \& Shi Y. Proposal for prevention and control of the 2019 novel coronavirus disease in newborn infants. Arch. Dis. Child Fetal Neonatal Ed. fetalneonatal-2020-318996 (2020).

69. Wang, J., Qi, H., Bao, L., Li, F. \& Shi, Y. A contingency plan for the management of the 2019 novel coronavirus outbreak in neonatal intensive care units. Lancet Child Adolesc. Health 4, 258-259 (2020).

70. Grasselli, G. et al. Baseline characteristics and outcomes of 1591 patients infected with SARS-CoV-2 admitted to ICUs of the Lombardy Region, Italy. JAMA 323, 1574-1581 (2020).

71. Guan, W. J. et al. Clinical characteristics of coronavirus disease 2019 in China. N. Engl. J. Med. 382, 1708-1720 (2020).

72. Mehta, P. et al. COVID-19: consider cytokine storm syndromes and immunosuppression. Lancet 395, 1033-1034 (2020).

73. Licciardi, F. et al. COVID-19 and what pediatric rheumatologists should know: a review from a highly affected country. Pediatr Rheumatol Online J 18, 35 (2020)

74. Li, H., Chen, K., Liu, M., Xu, H. \& Xu, Q. The profile of peripheral blood lymphocyte subsets and serum cytokines in children with 2019 novel coronavirus pneumonia. J. Infect. 81, 115-120 (2020).

75. Riphagen, S. G. X., Gonzalez-Matinez, C., Wilkinson, N. \& Theocharis, P. Hyperinflammatory shock in children during COVID-19 pandemic. Lancet 395, 16071608 (2020).

76. Jones, V. G. et al. COVID-19 and Kawasaki disease: novel virus and novel case. Hosp. Pediatr. 10, 537-540 (2020).

77. Li, W., Cui, H., Li, K., Fang, Y. \& Li, S. Chest computed tomography in children with COVID-19 respiratory infection. Pediatr. Radiol. 50, 796-799 (2020).

78. Feng, K. et al. [Analysis of CT features of 15 children with 2019 novel coronavirus infection]. Zhonghua Er Ke Za Zhi 58, 275-278 (2020).

79. Mungmunpuntipantip, R. \& Wiwanitkit, V. Chest computed tomography in children with COVID-19. Pediatr. Radiol. 50, 1018 (2020).

80. Denina, M. et al. Lung ultrasound in children with COVID-19. Pediatrics 146, e20201157 (2020).

81. Di Nardo, M. et al. A literature review of 2019 novel coronavirus (SARS-CoV2) infection in neonates and children. Pediatr Res (In Press, 2020). https://doi.org/ 10.1038/s41390-020-1065-5

82. ECMO in COVID-19. https://www.elso.org/COVID19.aspx.

83. Kneyber, M. C. J. et al. Recommendations for mechanical ventilation of critically ill children from the Paediatric Mechanical Ventilation Consensus Conference (PEMVECC). Intensive Care Med. 43, 1764-1780 (2017).
84. Cui, Y. et al. A 55-day-old female infant infected with COVID 19: presenting with pneumonia, liver injury, and heart damage. J. Infect. Dis. 221, 1775-1781 (2020).

85. Weiss, S. L. et al. Surviving Sepsis Campaign International Guidelines for the Management of Septic Shock and Sepsis-Associated Organ Dysfunction in Children. Pediatr. Crit. Care Med. 21, e52-e106 (2020).

86. Russell, C. D., Millar, J. E. \& Baillie, J. K. Clinical evidence does not support corticosteroid treatment for 2019-nCoV lung injury. Lancet 395, 473475 (2020).

87. Chen, Z. M. et al. Diagnosis and treatment recommendations for pediatric respiratory infection caused by the 2019 novel coronavirus. World J. Pediatr. 16, 240-246 (2020).

88. Chiotos, K. et al. Multicenter initial guidance on use of antivirals for children with COVID-19/SARS-CoV-2. J. Pediatr. Infect. Dis. Soc. piaa045 (2020).

89. Grein, J. et al. Compassionate use of remdesivir for patients with severe Covid19. N. Engl. J. Med. 382, 2327-2336 (2020).

90. Wang, Y. \& Zhu, L. Q. Pharmaceutical care recommendations for antiviral treatments in children with coronavirus disease 2019. World J. Pediatr. 16, 271274 (2020).

91. Kelvin, A. A. \& Halperin, S. COVID-19 in children: the link in the transmission chain. Lancet Infect. Dis. 20, 633-634 (2020).

92. Bedford, J. et al. COVID-19: towards controlling of a pandemic. Lancet 395 , 1015-1018 (2020).

93. World Health Organization. Coronavirus disease (COVID-19) outbreak: rights, roles and responsibilities of health workers, including key considerations for occupational safety and health. (2020). https://www.who.int/publications/i/ item/coronavirus-disease-(covid-19)-outbreak-rights-roles-and-responsibilitiesof-health-workers-including-key-considerations-for-occupational-safety-andhealth.

94. PICS. PICS and ICS Joint Position Statement (12 Mar 2020). https://picsociety.uk/ news/pics-and-ics-joint-position-statement-12-mar-2020/.

95. World Health Organization. Interim recommendations on obligatory hand hygiene against transmission of COVID-19. (2020) https://www.who.int/ publications/m/item/interim-recommendations-on-obligatory-hand-hygieneagainst-transmission-of-covid-19. Accessed $7^{\text {th }}$ May 2020

96. Kotecha, R. S. Challenges posed by COVID-19 to children with cancer. Lancet Oncol. 21, e235 (2020).

97. Wei, M. et al. Novel coronavirus infection in hospitalized infants under 1 year of age in China. JAMA 323, 1313-1314 (2020).

98. Forrester, J. D., Nassar, A. K., Maggio, P. M. \& Hawn, M. T. Precautions for operating room team members during the COVID-19 Pandemic. J. Am. Coll. Surg. 230, 1098-1101 (2020).

99. Zhou, Y., Xu, H., Li, L. \& Ren, X. Management for patients with pediatric surgical disease during the COVID-19 epidemic. Pediatr. Surg. Int 36, 751-752 (2020).

100. Yen, M. Y. et al. Interrupting COVID-19 transmission by implementing enhanced traffic control bundling: Implications for global prevention and control efforts. J. Microbiol. Immunol. Infect. 53, 377-380 (2020).

101. Woo Baidal, J. A. et al. Zooming towards a telehealth solution for vulnerable children with obesity during COVID-19. Obesity (Silver Spring) 28, 1184-1186 (2020).

102. Verstraete, S. G., Sola, A. M. \& Ali, S. A. Telemedicine for Pediatric Inflammatory bowel disease in the Era of COVID-19. J. Pediatr. Gastroenterol. Nutr. 70, e140 (2020).

103. Rockwell, K. L. \& Gilroy, A. S. Incorporating telemedicine as part of COVID-19 outbreak response systems. Am. J. Manag. Care 26, 147-148 (2020). 